\title{
An Evaluation of Iterative Reconstruction Strategies on Mediastinal Lesion Detection Using Hybrid Ga-67 SPECT Images
}

\author{
N. F. Pereira ${ }^{*}$ H. C. Gifford ${ }^{\dagger}$, P. H. Pretorius ${ }^{\dagger}$, T. Farncombe ${ }^{\ddagger}$, M. Smyczynski $^{\dagger}$, R. Licho ${ }^{\dagger}$, \\ P. Schneider ${ }^{\dagger}$, and M. A. King ${ }^{\dagger}$ \\ *Department of Physics, University of Massachusetts, Amherst, MA \\ $\dagger$ Division of Nuclear Medicine, University of Massachusetts Medical School, Worcester, MA \\ $\$$ Hamilton Health Sciences, Ontario, Canada
}

\begin{abstract}
Hybrid LROC studies can be used to more realistically assess the impact of reconstruction strategies, compared to those constructed with digital phantoms. This is because hybrid data provides the background variability that is present in clinical imaging, as well as, control over critical imaging parameters, required to conduct meaningful tests. Hybrid data is obtained by adding Monte Carlo simulated lesions to disease free clinical projection data. Due to Ga-67 being a particularly challenging radionuclide for imaging, we use Ga-67 hybrid SPECT data to study the effectiveness of the various correction strategies developed to account for degradations in SPECT imaging. Our data was obtained using GE-VG dual detector SPECT-CT camera. After determining a target lesion contrast we conduct pilot LROC studies to obtain a near-optimal set of reconstruction parameters for the different strategies individually. These near-optimal parameters are then used to reconstruct the final evaluation study sets. All LROC study results reported here were obtained employing human observers only. We use final LROC study results to assess the impact of attenuation compensation, scatter compensation and detector resolution compensation on data reconstructed with the RBI-EM algorithm. We also compare these with FBP reconstructions of the same dataset. Our experiment indicates an improvement in detection accuracy, as various degradations inherent in the image acquisition process are compensated for in the reconstruction process.
\end{abstract}

\section{Introduction}

Digital anthropomorphic phantoms have been extensively employed to simulate tomographic acquisitions via Monte Carlo methods for the purpose of studying relative performance of the reconstruction strategies [1]-[4]. Such simulation techniques provide an invaluable tool in studying the accuracy of a reconstruction algorithm, and in validating the effects of the various compensation strategies employed to correct for the various factors that degrade the image data acquisition process. They are, however, limited in their ability to provide a completely relevant assessment of the quality of reconstructions. This is because the background as provided by a mathematical phantom lacks the variability that is present in clinical imaging. While some effort has been made to capture this variability in simulated backgrounds [5], it might be more realistic to use disease free real patient backgrounds obtained in clinical studies and introduce into them simulated lesion activity [6]. Such hybrid images can be used for a more clinically realistic assessment of competing reconstruction algorithms and the various compensation strategies employed. 
We have conducted such a study. Our dataset consists of clinically obtained, disease-free Ga-67 SPECT/CT acquisitions, to which Monte-Carlo generated tumors have been added. We use Ga-67 citrate projection data, as this particular radionuclide emits a variety of energy photons which complicate attenuation and scatter correction and require the usage of medium energy collimators. Added to this, patient specific background variability further impacts lesion detection accuracy. Ga-67 data thus provides a rich test bed for studying the performance of the various reconstruction strategies. Our aim is to perform a systematic study of the impact of these reconstruction strategies on detection accuracy using hybrid data. We conduct human observer localization ROC (LROC) studies on the reconstructed images, to quantify and evaluate the impact of the different methods. The reconstruction strategies compared were: $i$. attenuation compensation (AC), ii. scatter compensation (SC) using the ESSE method, and iii. resolution compensation (RC) [7]-[12], using the RBI-EM iterative reconstruction algorithm. We compare these with FBP reconstructions of the same dataset [16]. Prior to comparison the parameter set of the reconstruction algorithms for each strategy have been optimized via preliminary LROC studies. We have chosen the mediastinal area as the region of interest for our investigation. This is because the mediastinum provides the most challenging task for Ga-67 citrate imaging clinically.

\section{Methods}

The work is subdivided into three parts $i$. hybrid data generation, ii. pilot LROC studies and iii. final evaluation study.

\section{A. Hybrid Data Generation}

The clinical datasets used for anatomical backgrounds consist of 11 disease free Ga-67 SPECTCT acquisitions obtained using the GE-VG SPECT/CT system. In all cases patient identities were masked from the datasets and image usage was under IRB approval. Each patient volume was then used to create 15 abnormal cases with one tumor per case. Individual volume slices were not used more than once. The lesions were created as 1-cm diameter spheres filled with uniform activity and were randomly placed within the clinically relevant mediastinal area of each patient. The mediastinal area was defined using patient specific masks obtained by segmenting the reconstructed CT images. Lesion projections were then generated with the Monte-Carlo program SIMIND [13] using attenuation weights supplied by the transmission maps. The tumor projections were then scaled relative to the background and added to it. A pilot LROC study employing human observers was conducted to determine the lesion scaling factor which produced an average area of $\approx 0.8$ under the LROC curve. The image sets used for this study were reconstructed using the RBI-EM [14] method with AC, RC and SC using the ESSE method [15] and used 15 RBI iterations with a subset of size 4. A Gaussian kernel having standard deviation $\sigma=1.0$ was used to smoothen the output data for these initial studies. For LROC studies, the image slices containing the lesion centers were then extracted, adaptively thresholded, interpolated to $256 \times 256$ and converted to 8 bit grey-scale format. The thresholding was performed in the following manner: the maximum lesion count values $(M L C)$ for all the images with lesions were evaluated. The maximum and the standard deviation of the $M L C$ 's were then determined. For a particular image, pixels having values greater than one standard deviation from the maximum of the $M L C$ value were set to the maximum $M L C$ value:

$$
I(x, y)= \begin{cases}\max (M L C) ; & I(x, y) \geq \max (M L C)+\sigma(M L C) \\ I(x, y) ; & \text { otherwise }\end{cases}
$$


Here, $I(x, y)$ is the pixel value of the image at $(x, y)$, and $\sigma(M L C)$ is the standard deviation of the $M L C$ values. Note that each LROC study session consisted of an initial training session followed by the test-study session. This was done to acclimatize the observer to the study environment.

\section{B. Pilot Study}

Human observer pilot studies were conducted using 3 of the 11 patient datasets to optimize the reconstruction parameters. In reality this can only produce a near-optimal parameter set. The optimizations were performed for each strategy individually. The strategies compared are $i$. RBI-EM with no correction (NC), ii RBI-EM with attenuation compensation (AC), iii. RBIEM with resolution compensation (RC), $v$. RBI-EM with AC and SC (ASC), $v$. RBI-EM with $\mathrm{AC}, \mathrm{SC}$ and RC (ALLC) and vi. FBP. For the iterative strategies the parameters optimized are the iteration number for the RBI-EM algorithm and the standard deviation of the post reconstruction Gaussian filter. Under FBP, the only parameter optimized is the cut-off frequency of the post-reconstruction Butterworth filter. Care was taken to randomize the reading order of the images for each study session, as well as the study order for each reader. The readers for this phase of the study consisted of three physicists and one physician.

\section{Final Evaluation}

Images for the final study set were reconstructed using the remaining 8 clinical datasets. There were 120 normal and 120 abnormal images. 40 of these images (20 normal and the corresponding 20 abnormal cases) were used for observer training at the start of each study session. The remaining 200 images were evaluated by each individual observer over two study sessions. The observer pool consisted of 6 readers ( 3 of whom were physicists and 3 physicians).

\section{Results And Discussions}

The near-optimal values for the iterative reconstructions are shown in Table I while that for the $\mathrm{FBP}^{1}$ reconstruction is shown in Table II. Table III displays the LROC ares obtained in the final evaluation studies. The areas were determined using Swensson's LROC software [18] applied to the individual observer response data. Prior to running the LROC software, we pool each observer's response data for a given strategy. The mean observer LROC area scores, shown in Table III were then computed.

Figure 1(a) and 1(b) respectively depict a background slice with and without the lesion present as reconstructed with the different strategies. Figures 2(a)-2(b) are box-plots ${ }^{2}$ of the observer LROC areas and standard errors for the strategies. The area plot indicates an overall difference between LROC scores corresponding to the strategies.

In order to assess the significance of these differences, a two-way ANOVA [19], [20] was performed on the LROC areas by categorizing the observer and strategy factors. Table IV displays the results of the analysis. Note that the $p$-values for the $F$ statistic across both factors are much lower than a 0.01 significance level. This indicates significant differences among the strategies and among observers.

Tukey's HSD [19] test was then employed to assess the significance of pairwise differences between two given strategies. The results of this test are displayed in TableV The test indicates that observer performance under the ALLC strategy is significantly greater $\alpha=0.05$ than the

\footnotetext{
${ }_{1}^{1}$ The filter order for the Butterworth filter was not optimized, however, we present it here for completeness.

${ }^{2}$ The plots were created using the statistical analysis package R [20]. Each box-plot contains the extreme of the lower whisker, the lower hinge, the median, the upper hinge and the extreme of the upper whisker for one group of data.
} 
other strategies. However, no significant pair-wise differences are detected between the RC, AC or ASC strategies, though, each of these strategies differ significantly with the NC and FBP strategies. Finally, observer performances under the NC and FBP are practically identical.

To assess any interactions effects that might be present between strategy, observer and the individual patient datasets, a 3-way ANOVA-with-interaction test was performed. In order to carry out this analysis, the LROC scores have to be categorized across 3 factors and the additional factor in this case is the individual patient dataset. Note, however, that as each patient dataset is used to generate a total of 30 images, the LROC areas computed using the response scores corresponding to each of the strategy-observer-dataset combination will not be robust. In order to circumvent this we use pseudo-values.

To compute pseudo-values, let $k$ denote the total number of datasets, let $Q_{k}$ be the LROC area computed using response scores from all datasets and take $Q_{i, k-1}$ to be the LROC area computed by excluding the response scores for the $i$-th dataset. The pseudo-value corresponding to the $i$-th dataset is then defined by:

$$
\tilde{Q}_{i}=k Q_{k}-(k-1) Q_{i, k-1}
$$

Pseudo-values are jack-knife transformations of the LROC areas, however, note that they can be negative.

Figure 3(a) is the Q-plot of the computed pseudo-values and indicates a reasonable adherence to normality. Figures 3(b), 3(c) and 3(d) are plots of the pseudo-values over the three factors. Table VI lists the means and standard deviations of the pseudo-values computed for each strategy. Note that the pseudo-value means are quite similar to the mean observer LROC areas shown in Table III.

The presence of 3 factors give rise to an equal number of two-way ANOVA-with-interaction tests. In our case only one of these three tests resulted in a significant pairwise interaction effect, the two interacting factors being strategy and dataset. Table.VII lists the ANOVA results. Figure 4(a) is the plot between strategy and observer factors plotted across different levels of the dataset factor. Note how the plot lines corresponding to different levels of dataset factor intersect appreciably, indicating interaction. Figure 4(b) is the interaction plot between the observer-dataset factors, and has been included to convey an idea of factors that do no interact significantly.

Figure 5(a) displays slices from the different patient datasets reconstructed using RBI-EM with attenuation and scatter compensation. The images convey an idea of the background variability that an observer deals with when evaluating images. A 3-way ANOVA was also carried out on the pseudo-value dataset with the same 3 factors. The results are shown in Table VIII. Apart from detecting overall significant differences arising due to strategy and observer variability, the 3-way ANOVA also detects significant differences that arise due to variability in the reconstructed patient dataset. The plots in Figure 3(c) visually convey this.

\section{Conclusions}

We have completed a systematic study on the human observer detection performance of the RBI-EM iterative reconstruction algorithm with various compensation strategies included. Analysis of the observer response data indicates the following: 
- Post ANOVA analysis detects significant pairwise differences in detection performance between the RBI-EM with the all corrections strategy and each of the other strategies. The all correction strategy has superior detection performance in each case.

- No significant difference in detection performance is detected between RBI-EM reconstructed with $\mathrm{RC}, \mathrm{AC}$ or $\mathrm{AC}+\mathrm{SC}$. However each of these strategies have superior performance when compared to the RBI-EM with no corrections and with the FBP reconstruction method.

- No significant difference in performance is detected between RBI-EM with no corrections and the FBP reconstruction strategies.

- ANOVA analysis indicates significant inter-observer performance differences (Figure 3(d)) as well as, differences in performance due to variability in reconstructed patient datasets (Figure 3(c)).

- Significant interaction effects between reconstruction strategy and patient datasets could be due the parameter optimization scheme used in the study. To recapitulate, for the RBI-EM algorithm, we optimized the iteration number and the postreconstruction Gaussian blur, while for the FBP method, the cut-off frequency of the post-reconstruction Butterworth filter was optimized. Moreover, optimizations were performed using a portion of the patient background which were not re-used for the evaluation studies. However, there is no firm reason to believe that parameters optimized for a subset of the data should be optimal for the entire set, and it is quite plausible that detection performance across strategies will be different if individual patient datasets could be optimized separately.

- Detection accuracy, as measured via human observer LROC areas, tend to improve as more compensation strategies are incorporated into the reconstruction process. The standard deviation in the LROC areas/pseudo-values for a given strategy, computed over reader-dataset combinations, can be considered to be a measure of the reconstruction noise associated with that strategy. The decreasing value of the standard deviation, as more corrections are incorporated (see Table VI), therefore indicates an improvement in image quality.

\section{Acknowledgements}

This work was supported by National Institute for Biomedical Imaging and Bioengineering under grant R01-EB02798. The contents are solely the responsibility of the authors and do not necessarily represent the official views of the National Institute of Health. The authors would also like to thank Lily Krnezich for assistance in acquiring the clinical studies.

\section{References}

1. Gifford HC, King MA, Narayanan MV, Pretorius PH. Effect of block-iterative acceleration on Ga-67 tumor detection in thoracic SPECT. IEEE Trans Nucl Sci 2002;49:50-55.

2. Gifford HC, Wells RG, King MA. A comparison of human observer LROC and numerical observer ROC for tumor detection in SPECT images. IEEE Trans Nucl Sci 1999;46:1032-1037.

3. Gifford HC, King MA, Pretorius PH, Wells RG. A comparison of human and model observers in multislice LROC studies. IEEE Trans Med Img 2005;24:160-169.

4. Narayanan MV, Gifford HC, King MA, Pretorius PH, Farncombe TH, Bruyant P, Wernick MN. Optimization of iterative reconstructions of $T c^{99 m}$ cardiac SPECT studies using numerical observers. IEEE Trans Nucl Sci 2002;49:2355-2360. 
5. Rolland, JP. Synthesizing anatomical images for image understanding. In: Van Metter, R.; Beutel, J.; Kundel, H., editors. Handbook of Medical Imaging: Volume 1, Progress in Medical Physics and Psychophysics. SPIE Press; 2000. p. 685-713.

6. Zheng XM, Gifford HC, Pretorius PH, King MA. An observer study of reconstruction strategies for the detection of solitary pulmonary nodules using hybrid Neotect SPECT images. IEEE Nucl Sci Symp and Med Imaging Conf Portland, OR, USA. 2003

7. Tsui BMW, Hu HB, Gilland DR, Gullberg GT. Implementation of simultaneous attenuation and detector response correction in SPECT. IEEE Trans Nucl Sci 1988;35:778-783.

8. Liang Z, Turkington TG, Gilland DR, Jaszczak RJ, Coleman RE. Simultaneous compensation for attenuation, scatter and detector response for SPECT in three dimensions. Phy Med Biol 1992;37:587603.

9. King MA, Glick SJ, Pretorius PH, Wells RG, Gifford HC, Narayanan MV, Farncombe T. Attenuation, Scatter and Spatial Resolution Compensation in SPECT. IEEE Proc Conf on Comp Vis Patt Recog. 1987

10. Gifford HC, King MA, Wells RG. LROC analysis of detector-response compensation in SPECT. IEEE Trans Med Img 2000;19:463-473.

11. Gifford HC, King MA, Wells RG, Hawkins WG, Narayanan MV, Pretorius PH. LROC analysis of detector-response compensation in SPECT. Proc Fully 3D Img Recon in Rad Nucl Med 1999:8083.

12. Farncombe TH, Gifford HC, Narayanan MV, Pretorius PH, Frey EC, King MA. Assessment of scatter compensation strategies for Ga-67 SPECT using numerical observers and human LROC studies. J Nucl Med 2004;45:802-812. [PubMed: 15136630]

13. Ljungberg, M. The SIMIND Monte-Carlo program. In: Ljungberg, M.; Strand, SE.; King, MA., editors. Monte-Carlo calculation in Nuclear Medicine. IOP Publishing; Bristol and Philadelphia: p. 145-163.

14. Byrne CL. Block iterative methods for image reconstructions from projections. IEEE Trans Img Proc 1996;5:792-794.

15. Frey EC, Tsui BMW. A new method for modeling the spatially-variant, object-dependent scatter response function in SPECT. IEEE Nucl Sci Symp and Medical Imaging Conf, Anaheim CA 1996:1082-1086.

16. Wells RG, King MA, Simkin PH. Comparing filtered back-projection and ordered-subsets expectation maximization for small lesion detection and localization in Ga-67 SPECT. J Nucl Med Img 2000;41:1391-1399.

17. Gifford HC, King MA. Case Sampling in LROC: A Monte Carlo Analysis. Proc SPIE 4324:143152.

18. Swensson RG. Unified measurement of observer performance in detecting and localizing target objects on images. Med Phys 1996;10:1709-1725. [PubMed: 8946368]

19. Myers JL, Wells AD. Research Design and Statistical Analysis. Lawrence Erlbaum Associates. 2002

20. Dalgaard P. Introductory Statistics with R. Springer. 2002 


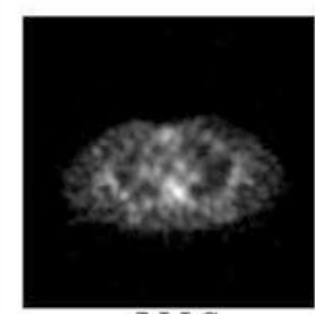

ALLC

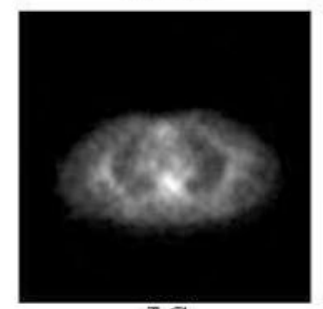

$\mathrm{AC}$

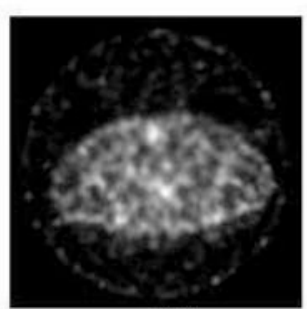

$\mathrm{RC}$

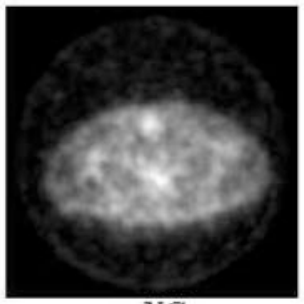

$\mathrm{NC}$

(a)

(b)

Fig. 1. Reconstructed background with lesion

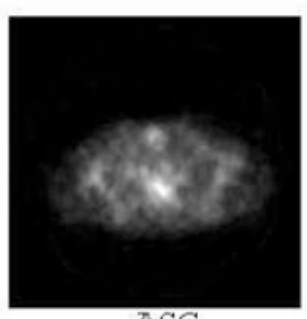

$\overline{A S C}$

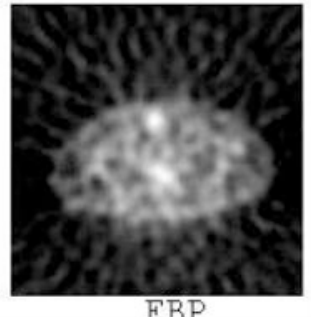

FBP

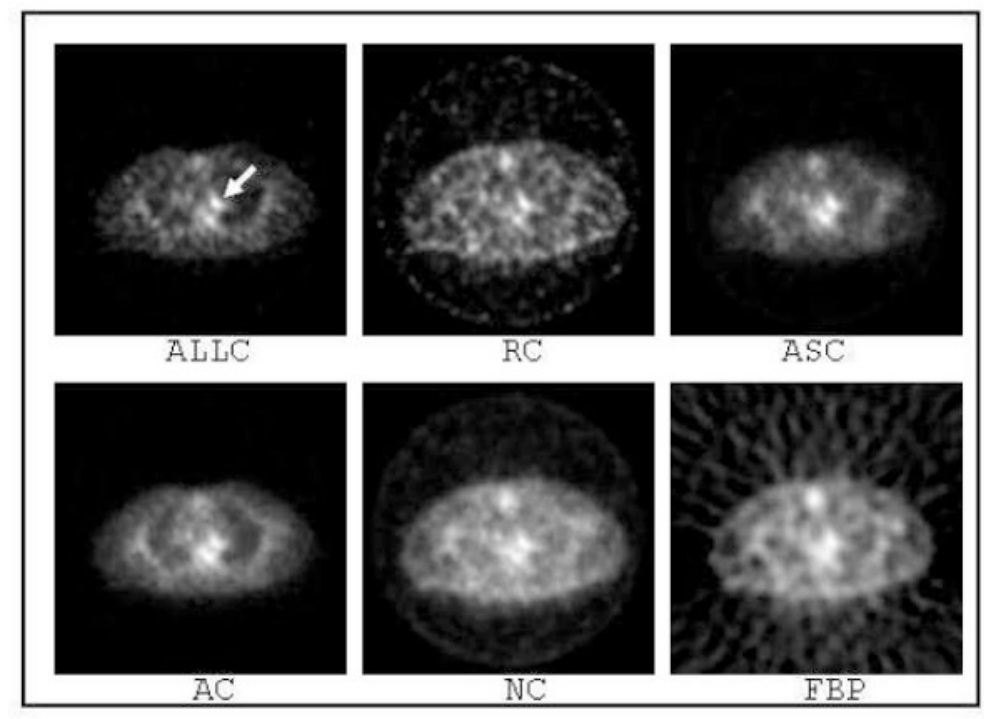

Data reconstructed under the different strategies: a. Reconstructed background, $b$. 


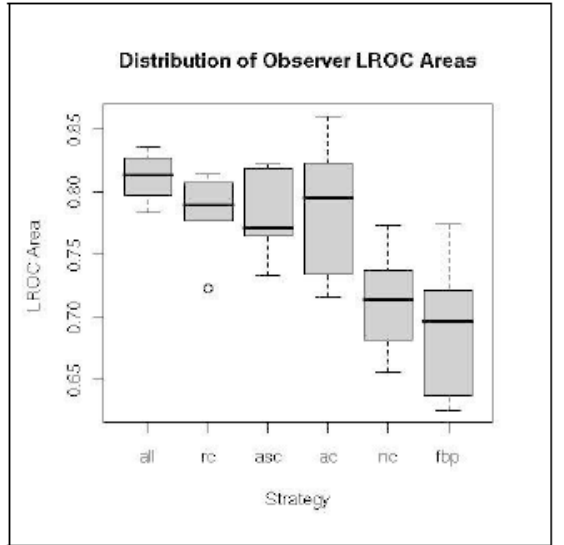

(a)

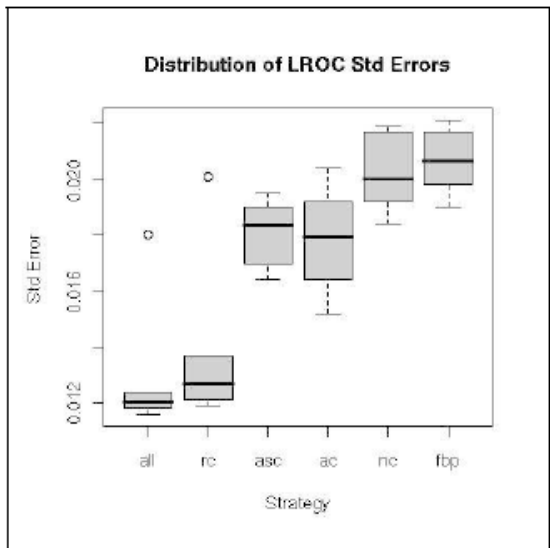

(b)

Fig. 2.

Relevant observer performance statistics under the strategies: a. Observer LROC areas, b. Standard errors of observer LROC areas 


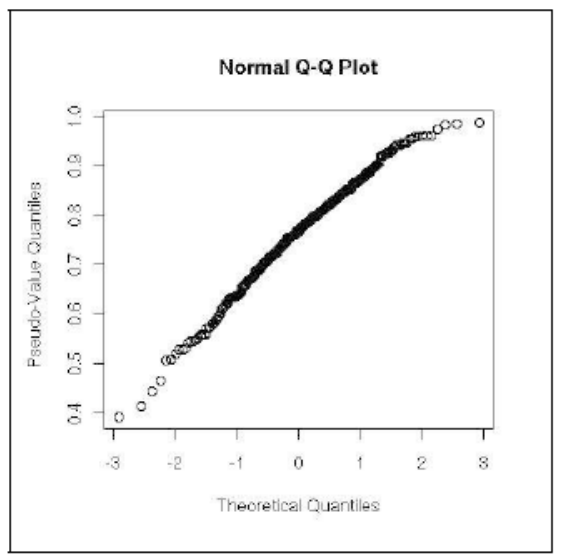

(a)

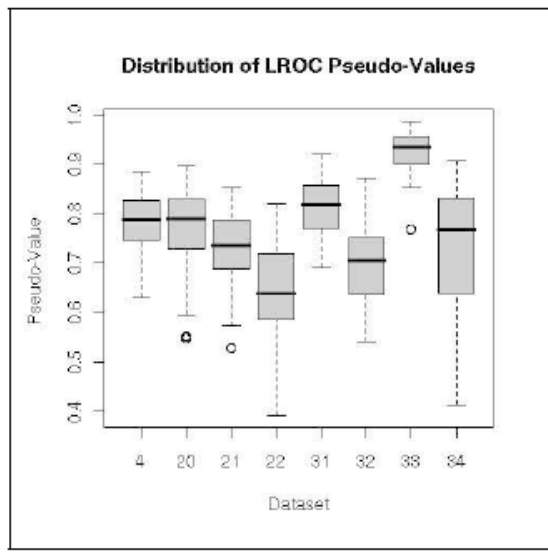

(c)

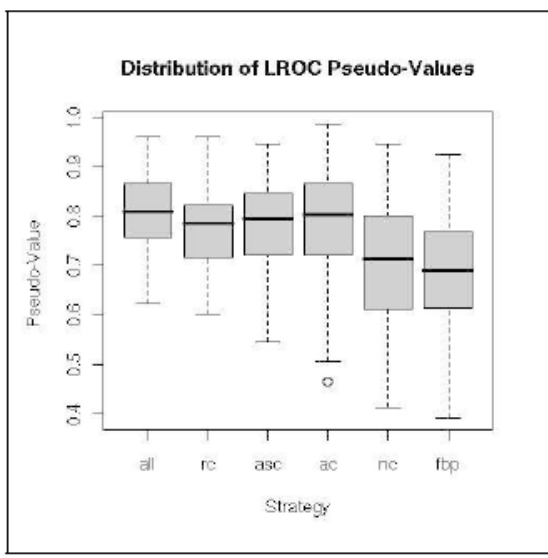

(b)

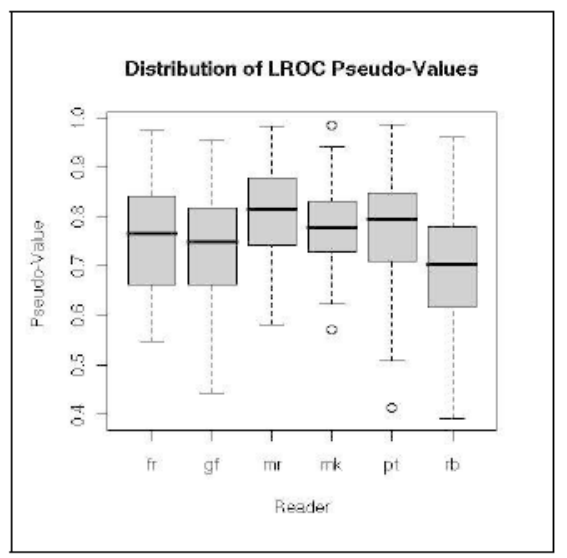

(d)

Fig. 3.

LROC Pseudo-Values: a. Pseudo-Values Q-plot, b. Pseudo-Values across Strategies, c. Pseudo-Values across Datasets, d. Pseudo-Values across Observers 


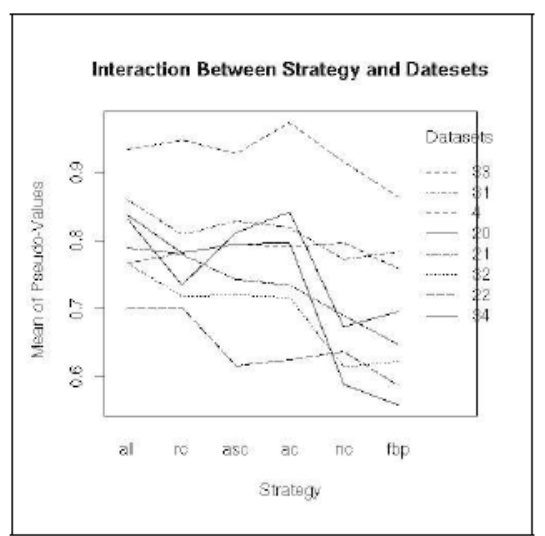

(a) Strategy-Dataset Interaction

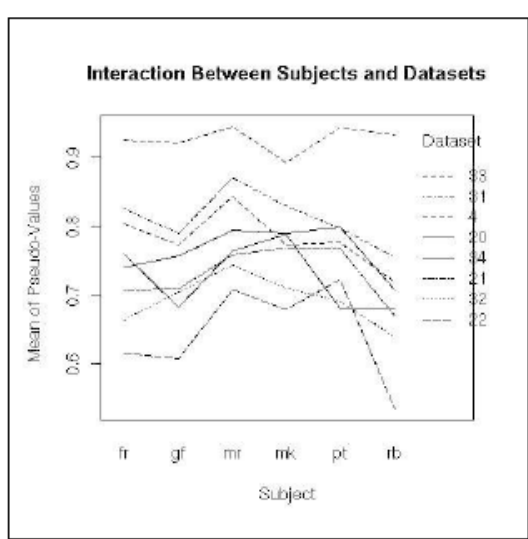

(b) Observer-Dataset Interaction

Fig. 4.

Interaction Between Factors 


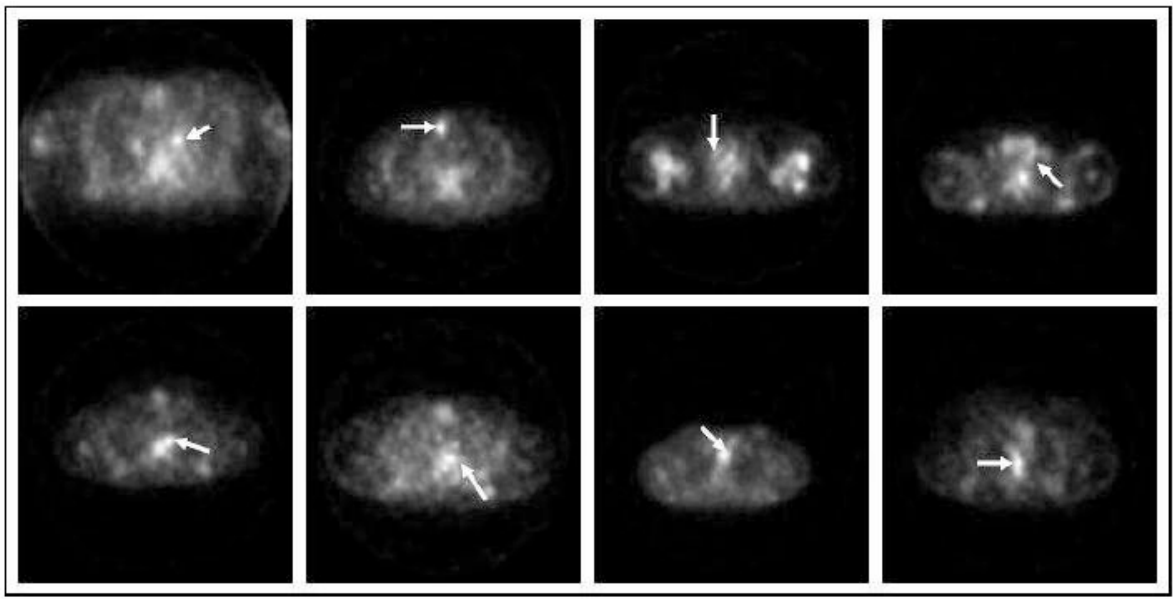

(a) Background with Lesion Reconstructions Corresponding to Different Patient Datasets

Fig. 5.

Strategy-Dataset Interactions 
Near-Optimal Parameters (Iterative)

\section{TABLE I}

\begin{tabular}{|c|c|c|}
\hline Strategy & Iteration & Blur(pix) \\
\hline All & 15 & 1.0 \\
\hline ASC & 3 & 1.25 \\
\hline AC & 3 & 1.25 \\
\hline RC & 15 & 1.0 \\
\hline NC & 5 & 1.5 \\
\hline
\end{tabular}




\section{TABLE II}

Near-Optimal Parameters (FBP)

\begin{tabular}{|r|r|c|}
\hline Strategy & Filter Order & Cut-off Frequencv \\
\hline FBP & 5 & 0.14 \\
\hline
\end{tabular}




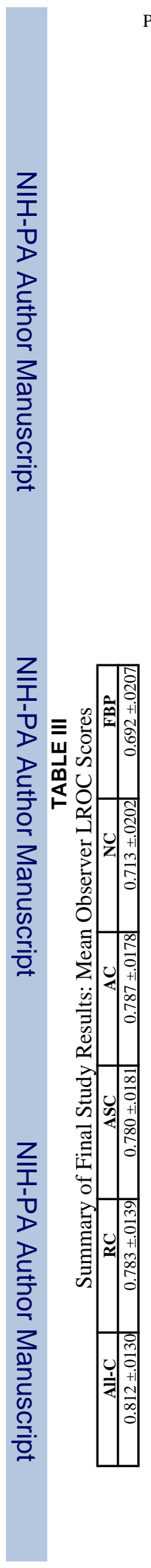




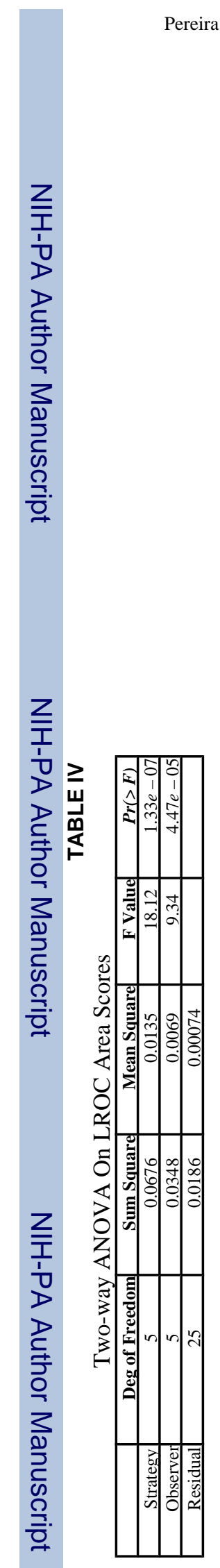

Page 15

IEEE Nucl Sci Symp Conf Rec (1997). Author manuscript; available in PMC 2009 January 23. 


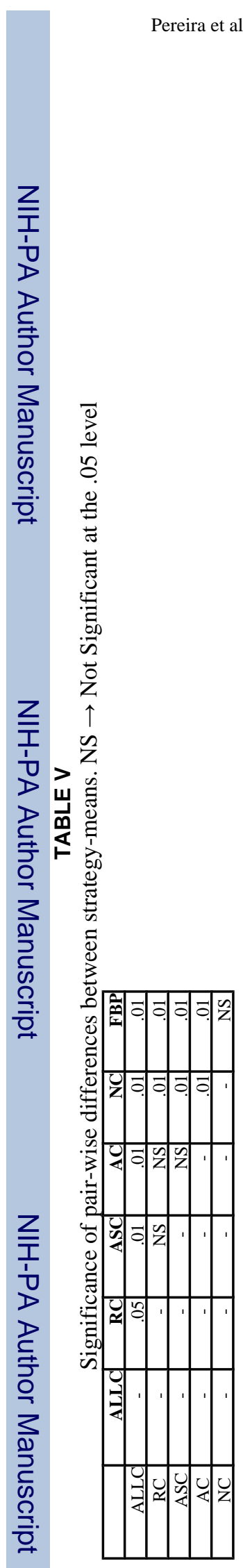

IEEE Nucl Sci Symp Conf Rec (1997). Author manuscript; available in PMC 2009 January 23. 


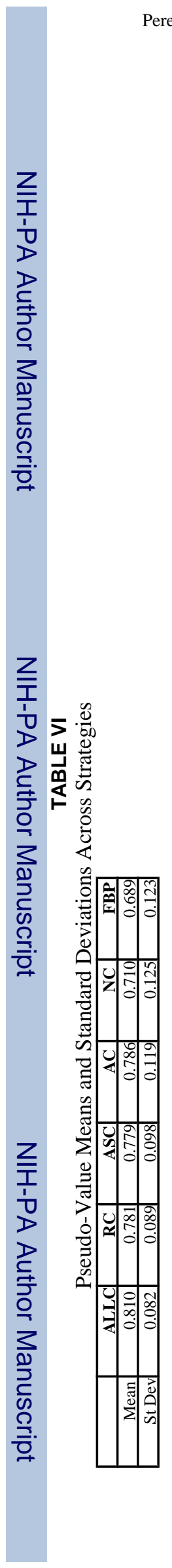




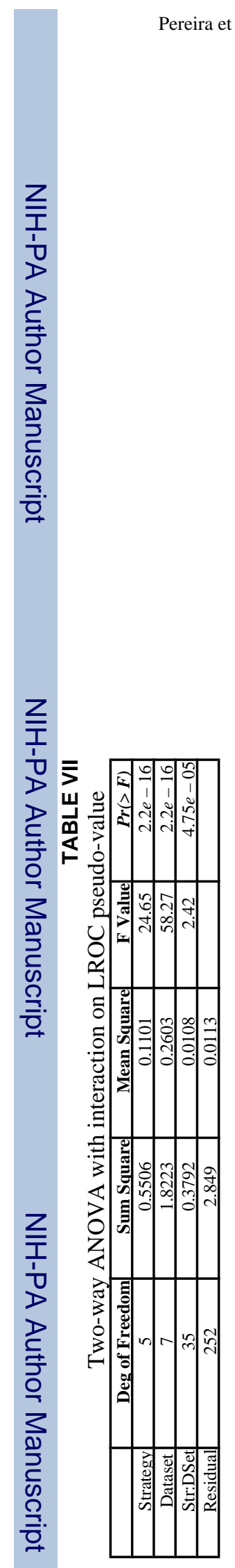

IEEE Nucl Sci Symp Conf Rec (1997). Author manuscript; available in PMC 2009 January 23. 


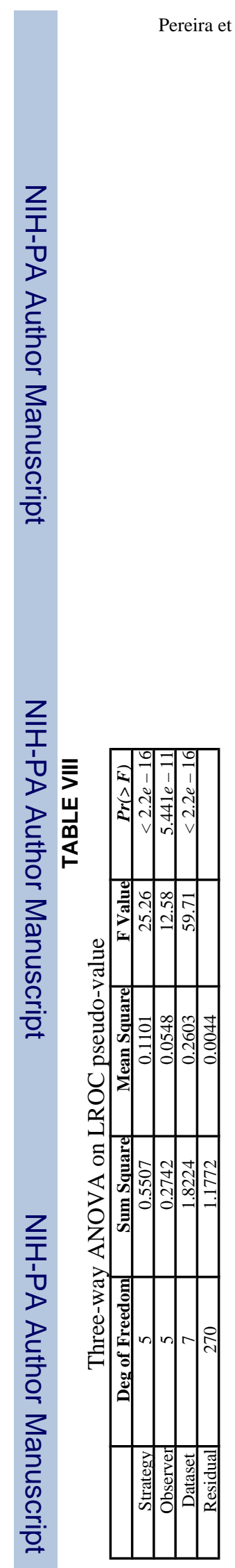

Page 19

IEEE Nucl Sci Symp Conf Rec (1997). Author manuscript; available in PMC 2009 January 23. 